\title{
Modelling the maintenance of male-fertile cytoplasm in a gynodioecious population
}

\author{
M Dufaÿ ${ }^{1}$, P Touzet $^{1}$, S Maurice ${ }^{2}$ and J Cuguen ${ }^{1}$ \\ ${ }^{1}$ Laboratoire de Génétique et Evolution des Populations Végétales, UMR-CNRS 8016, FR CNRS 1818, Université des Sciences et \\ Technologies de Lille-Lille I, Villeneuve d'Ascq Cedex, France and 'Institut des Sciences de l'Evolution de Montpellier, UMR-CNRS \\ 5554, Université de Montpellier II, Place Eugène Bataillon, Montpellier Cedex, France
}

\begin{abstract}
Gynodioecy is the co-occurrence of females and hermaphrodites in populations. It is usually due to the combined action of cytoplasmic male sterility (CMS) genes and nuclear genes that restore male fertility. According to previous theoretical studies, it is very difficult to explain the maintenance of gynodioecy with CMS and male-fertile cytotypes, although it has been observed in some species. However, only very specific situations have been investigated so far. We present a model to investigate the conditions that promote the maintenance of this breeding system in the case of an outcrossed species when CMS and male-fertile (non-CMS) cytotypes are present in an infinite panmictic population. We show that the type of cost of restoration
\end{abstract}

strongly affects the conditions for stable maintenance of gynodioecy. Stable nuclear-cytoplasmic gynodioecy requires a female advantage, which is a classical condition for gynodioecy, but also a cost of CMS for female fitness, which had been rarely investigated. A cost of restoration is also needed, which could affect either pollen or seeds. Finally, we found that gynodioecy was attainable for a large set of parameter values, including low differences in fitness among genotypes and phenotypes. Our theoretical predictions are compared with previous theoretical work and with results of empirical studies on various gynodioecious species.

Heredity (2007) 99, 349-356; doi:10.1038/sj.hdy.6801009; published online 6 June 2007

Keywords: gynodioecy; cytoplasmic male sterility; model; female advantage; CMS cost; restorer cost

\section{Introduction}

Gynodioecy is a common plant sexual system, in which females and hermaphrodites co-occur in natural populations. This mating system is generally determined by both nuclear and cytoplasmic factors: cytoplasmic genes cause male sterility (CMS for cytoplasmic male sterility) while nuclear genes (restorers) can restore male fertility (Budar et al., 2003; Hanson and Bentolila, 2004; Chase, 2007). A number of experimental and theoretical studies have attempted to determine the conditions for the stable maintenance of gynodioecy. CMS can spread in populations when females benefit from a higher female fitness through resource reallocation, an effect termed female advantage or compensation (Darwin, 1877). Alternatively, the avoidance of inbreeding can also promote the spread of CMS (Charlesworth, 1981). In addition, stable nuclear-cytoplasmic gynodioecy requires either the negative pleiotropic effect of restorer alleles (cost of restoration; Charlesworth, 1981; Delannay et al., 1981; Frank, 1989; Gouyon et al., 1991; Bailey et al., 2003) or a meta-population structure with local extinction and restricted gene flow, which renders the cost of restoration unnecessary (Pannell, 1997; Couvet et al., 1998; McCauley et al., 2000). Previous theoretical studies have shown that,

Correspondence: Dr M Dufä̈, Laboratoire de Génétique et Evolution des Populations Végétales, UMR-CNRS 8016, FR CNRS 1818, Université des Sciences et Technologies de Lille-Lille I, 59655 Villeneuve d'Ascq Cedex, France.

E-mail: mathilde.dufay@univ-lille1.fr

Received 23 October 2006; revised 14 April 2007; accepted 25 April 2007; published online 6 June 2007 under certain conditions, gynodioecy can be maintained with limit cycles (undampened oscillations of female frequencies) that could explain the large variation in female frequencies observed among gynodioecious populations of a single species (Gouyon et al., 1991; Bailey et al., 2003).

Models that have been published to explain gynodioecy vary in terms of (i) the population structure assumed, which can be either an infinite panmictic population or a meta-population; (ii) the genetic determination of sex, in particular whether it includes only one or several sterile cytotypes; (iii) how differences in fitness among genotypes (female advantage and cost of restoration) are expressed and (iv) the degree of selfing of the hermaphroditic individuals and the possibility of pollen limitation. It must be noted that selfing can have an important impact on gynodioecy in two opposite ways: females can be favoured if inbreeding depression occurs, but if female frequencies reach high values, pollen may become limited and hermaphrodites that partly reproduce by selfing may be then favoured (McCauley and Taylor, 1997). This can induce frequency-dependent selection between the sexes, and consequently between cytotypes (Lewis, 1941; Charlesworth, 1981) promoting the maintenance of gynodioecy. This last explanation is obviously inadequate in the case of self-incompatible species, in which hermaphrodites cannot gain an advantage during pollen shortages by self-fertilization.

Most of the theoretical studies on gynodioecy have focused on situations in which all cytotypes are malesterile, which is effectively the case of some well-studied 
gynodioecious species such as Silene vulgaris (Olson and McCauley, 2002), Silene acaulis (Städler and Delph, 2002) and Thymus vulgaris (Belhassen et al., 1991). However, male-fertile cytotypes have been reported in two selfincompatible gynodioecious species: Plantago lanceolata (De Haan et al., 1997b) and Beta vulgaris ssp. maritima (Cuguen et al., 1994). In the latter species, the wild sea beet, non-sterilizing cytotypes constitute a large part of the mitochondrial diversity (Cuguen et al., 1994; Forcioli et al., 1998; Laporte et al., 2001). Moreover, the most frequent cytoplasm is male-fertile and because the four different CMS cytotypes are derived from it, this malefertile cytotype is likely to be ancestral (Fénart et al., 2006). In other species, male-fertile cytotypes are a common feature of gynodioecious agricultural plants such as maize (Fauron and Casper, 1994; Clifton et al., 2004), Brassica napus (Brown, 1999), or Sorghum (Tang et al., 1996). Finally, because of the lack of CMS markers or reciprocal cross data (for example, Van Damme et al., 2004), the occurrence of male-fertile cytotypes is usually not detectable. Thus, except for species for which all cytotypes, detectable through genetic markers, have been associated with male sterility (for example, Silene vulgaris, Olson and McCauley, 2002; Silene acaulis, Städler and Delph, 2002), or for which reciprocal crosses have been performed for all cytotypes, the occurrence of malefertile cytotypes in gynodioecious species cannot be rejected. It must be emphasized here that male-fertile cytoplasms could include a cytoplasm that does not produce sterilizing factors (that is, a 'normal' cytoplasm), or a sterilizing cytoplasm for which the nuclear restorer genes are fixed.

The most recent and complete published models on the maintenance of gynodioecy assumed only two cytotypes, both being CMS (Gouyon et al., 1991; Couvet et al., 1998; Bailey et al., 2003). In particular, Bailey et al. (2003) suggested that male-fertile cytotypes could not be maintained under the model's conditions: in these models, male-fertile cytotypes are never associated with a female phenotype and thus never benefit from female compensation, causing male-fertile cytotype to be at a selective disadvantage compared to CMS. On the other hand, Delannay et al. (1981) and Charlesworth (1981) have examined the coexistence of a sterile and a fertile cytotype, but they mainly focused on self-compatible species. Charlesworth (1981) briefly considered the case of purely out-crossing species and suggested also that the maintenance of cytoplasmic polymorphism in this case was not possible: when hermaphrodites lose the advantage of self-pollination the condition of spread of CMS are also the conditions of its fixation. However, Ross and Gregorius (1985) found that CMS and malefertile cytotypes could be maintained without obligate self-pollination, by adding a selective disadvantage in terms of female fitness to the nuclear restorer. Therefore, until now the conditions allowing the maintenance of both CMS and male-fertile cytotypes have appeared to be very restrictive, yet only a very limited set of situations has actually been investigated.

Bailey et al. (2003) showed that the type of cost of restoration can have a considerable influence on the gynodioecy maintenance when two male-sterile cytotypes co-occur. In contrast, the general conditions for the maintenance of gynodioecy with a male-fertile cytotype, particularly under various types of restoration costs, remain an open question. In this study, we will enlarge the set of conditions previously investigated by considering all possible types of restoration costs. In addition, we will focus on the less-studied case of species without selfing, for which gynodioecy has been suggested to be theoretically more complex to maintain. Because several gynodioecious plants are self-incompatible, this model may help to unravel the mechanisms maintaining gynodioecy in these species. We will not consider the effects of drift and gene flow, and will only focus on the role of selection.

\section{The model}

We considered a diploid, out-crossed species with nonoverlapping generations and based our recurrence equations closely on those presented by Gouyon et al. (1991). However, we initialized our model with one CMS and one male-fertile cytotype (instead of two CMSs). We further assumed that there were two alleles at the restorer locus, a dominant allele, restorer of male fertility and a recessive allele that maintained male sterility (see Table 1). For each generation, the gametic frequencies were calculated by adding the contributions of each genotype, using its frequency, its female and its male fitness (see below) and Mendelian segregation ratios. Zygote frequencies for the next generation were then calculated based on the ovule and pollen frequencies, assuming random mating.

We based our model on three fitness parameters: (1) a female advantage $F A$ that induces a higher seed production for females compared to hermaphrodites; this advantage is usually thought to be the result of a tradeoff between seed and pollen production; (2) a cytotype effect acting on female fitness named $w_{s}$, considering that this effect could either be positive or negative for the CMS; we then used the parameter $w_{s}$ to define female fitness of individual plants bearing CMS and (3) a cost of restoration $c$, which affects either pollen or seed. As in Bailey et al. (2003), three possible expressions were considered for the cost of restoration: (i) a constitutive cost which is cytoplasm-independent, (ii) a 'silent' cost (called 'alien' in Gouyon et al., 1991), for which only restorers that do not affect sex determination (that is, on a male-fertile cytotype) incur a cost and (iii) an expressed cost, concerning only restorers on a male-sterile cytotype. Both dominant and recessive costs were considered. This then defined 12 different types of restorer cost, which were studied in 12 different models (Figure 1). Fitness of all genotypes was a combination of these three parameters and in particular the female fitness of male-sterile

Table 1 Relationships between nucleo-cytoplasmic genotype and sexual phenotype

\begin{tabular}{llll}
\hline Cytotype & \multicolumn{3}{c}{ Restorer locus } \\
\cline { 2 - 4 } & $m m$ & $m R$ & $R R$ \\
\hline CMS & Female & Herma. & Herma. \\
Male-fertile & Herma. & Herma. & Herma. \\
\hline
\end{tabular}

Abbreviation: CMS, cytoplasmic male sterility.

Two alleles occur at the restorer locus: the maintainer of male sterility $(m)$ and the restorer of male fertility $(R)$. Hermaphrodites with CMS are then called restored hermaphrodites. 
plants was the product of the CMS effect and the female advantage. An example of pollen and seed fitness expressions is detailed in Table 2.

This modelling work was limited to the following initial condition: $99 \%$ of individuals have a male-fertile cytotype and two recessive maintainer alleles at the restorer locus while $1 \%$ of individuals are restored hermaphrodites (CMS, heterozygote at the restorer locus), simulating the arrival of restored hermaphrodite in a non-gynodioecious population. We examined the other possible initial conditions (simultaneous arrival of CMS and restorer in distinct individuals; arrival of restored hermaphrodites homozygous for the restorer allele). However, these alternate scenarios provided the

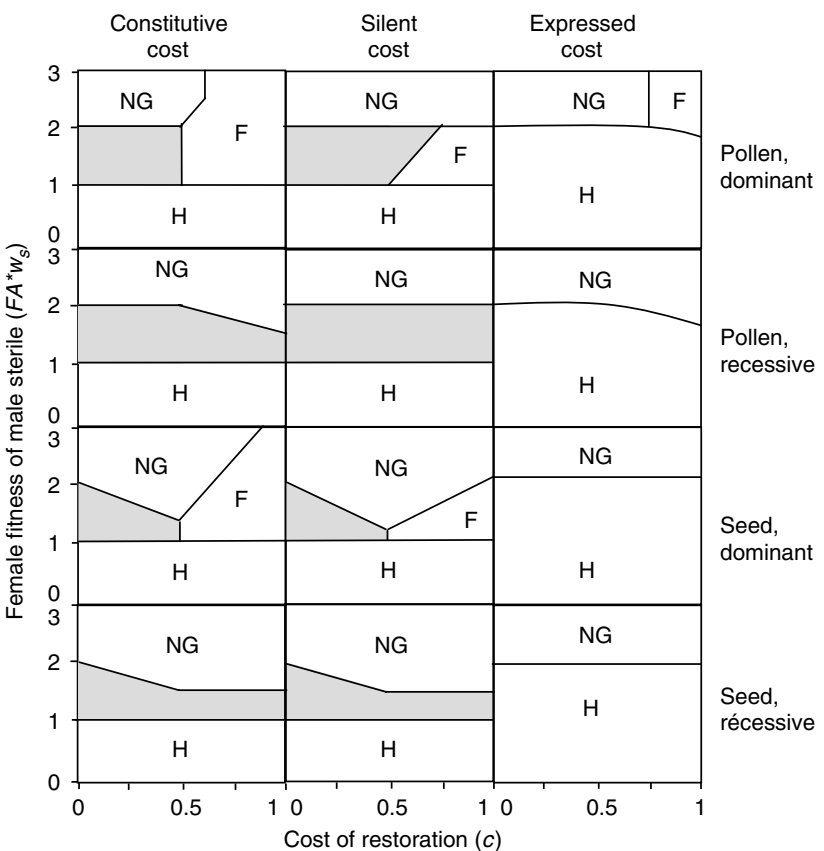

Figure 1 States of the simulated populations according to fitness parameter values in all 12 models. Regions where nuclearcytoplasmic gynodioecy was maintained are displayed in grey. Other cases included nuclear gynodioecy (NG), for which the CMS invaded and some polymorphism at the restorer locus was maintained, reversion to hermaphroditism $(\mathrm{H})$ and fixation of non-restored CMS, leading to a population only composed of females $(\mathrm{F})$

Table 2 Seed fitness (upper part) and pollen fitness (lower part) expressions used in the model

\begin{tabular}{|c|c|c|}
\hline \multirow[t]{2}{*}{ Cytotype } & \multicolumn{2}{|c|}{ Restorer locus } \\
\hline & $m m$ & $m R$ and $R R$ \\
\hline CMS & $F A^{*} w$ & $(-c)$ \\
\hline Male-fertile & $1>1$ & $(-c)$ \\
\hline
\end{tabular}

Abbreviation: CMS, cytoplasmic male sterility.

This shows one particular case, where a constitutive cost of restoration acts on pollen and is dominant. same results at equilibrium and are therefore not presented. Deterministic computer calculations were run for all combinations of parameters with $F A \in[0 ; 5]$, $w_{s} \in[0 ; 2]$ and $c \in[0 ; 1]$, in steps of 0.05 . With regard to $w_{s}$ parameter values less than 1 represent a fitness cost to individuals with CMS cytotypes compared to individuals with male-fertile cytotypes; in other cases, CMS is directly advantageous to the plants whatever their sexual phenotype (female or restored hermaphrodite). Female fitness of females was then a combination of both the CMS effect and the female advantage $\left(F A^{*} w_{s}\right)$. Around borderlines, we explored parameter values on a finer scale (steps of 0.01). Simulations were run for at least 3000 generations. For each calculation, we considered nuclear-cytoplasmic gynodioecy to occur when both female and CMS frequencies fell between 0.02 and 0.98 . We recorded cases of both stable gynodioecy and limit cycles (in which case we recorded the maximum frequency of females in the cycle).

\section{Results}

Four main scenarios were revealed by the computer calculations: hermaphroditism (CMS cannot spread), nuclear gynodioecy (fixation of the CMS, and polymorphism at the restorer locus allows sexual polymorphism), female populations (fixation of the CMS and loss of the restorer allele, such populations are of course destined to extinction) and nuclear-cytoplasmic (NC) gynodioecy (polymorphism at both the restorer locus and the cytotype). The first three scenarios are not relevant for understanding the co-occurrence of malesterile and male-fertile cytotype because only one cytotype remained at equilibrium. We therefore focus only on the last, NC gynodioecy, which was found in 8 of the 12 models, for a portion of the parameter space. For completeness, we graphically present under what conditions the four scenarios (hermaphroditism, nuclear gynodioecy, female population and NC gynodioecy) were found in all 12 models in Figure 1.

\section{Effect of CMS and female advantage on gynodioecy maintenance}

In every model examined, NC gynodioecy was only found for $\left.w_{s} \in\right] 0 ; 1[$, that is, when CMS brought a fitness cost, and for values of female advantage $F A$ for which the product of female advantage and CMS effect $\left(F A^{*} w_{s}\right)$ was between 1.0 and 2.0. Because $F A^{*} w_{s}$ is the relative female fitness of male-sterile plants compared to non-CMS hermaphrodites, values higher than 1 were cases for which the female advantage compensated for the CMS cost and provided a selective advantage to male-sterile plants. When values of $F A^{*} w_{s}$ exceeded 2, the frequency of CMS increased rapidly in comparison with the restorer allele, and spread to fixation, which amounts to the situation of nuclear gynodioecy. As a consequence, we only found NC gynodioecy when restored hermaphrodites had the lowest female fitness (because of CMS cost), females had the highest female fitness and normal hermaphrodites were intermediate.

Effect of cost of restoration on gynodioecy maintenance $\mathrm{NC}$ gynodioecy was never found with an expressed cost of restoration (that is, affecting only restored hermaph- 
rodites, either on their pollen or seed production). For constitutive and silent dominant costs affecting pollen, $\mathrm{NC}$ gynodioecy was only found when cost values did not exceed 0.5 and $1 / 2^{*}\left(F A^{*} w_{s}-3 / 8\right)$, respectively (Figure 1 ). This last equation was found by performing a linear regression on simulations results. For higher cost values, the restorer could not be selected and simulations showed fixation of the non-restored CMS (females). In both cases (silent or constitutive), recessive costs of restoration allowed NC gynodioecy in a larger area of parameter space than did dominant ones (Figure 1): individuals that were heterozygous at the restorer locus did not have any cost, and this was sufficient to prevent fixation of CMS even for high cost values. Finally when cost affected seed production, NC gynodioecious situations were less frequent. But the major difference between these two situations (cost on pollen versus seed) concerned NC gynodioecy dynamics (see below).

\section{Dynamics of gynodioecy}

When NC gynodioecy was stable, results could be divided into two cases: either frequencies of CMS and females converged towards stable values, or frequencydependent selection resulted in regular variation of frequencies over time (limit cycles, Figure 2a). Limit cycles were only found for dominant cost and mainly concerned costs acting on pollen, for which about onethird of gynodioecious simulated populations displayed limit cycles. In contrast, when cost affected seed, a point equilibrium was reached for more than $95 \%$ of cases. In situations for which a limit cycle was not observed, point equilibria were reached after a dampened cycle, and it generally took at least several hundred generations for the equilibrium to be reached (Figure $2 b$ ). Therefore, in almost all cases, frequencies of genotypes and phenotypes showed high variation at least during the first step of the dynamics.
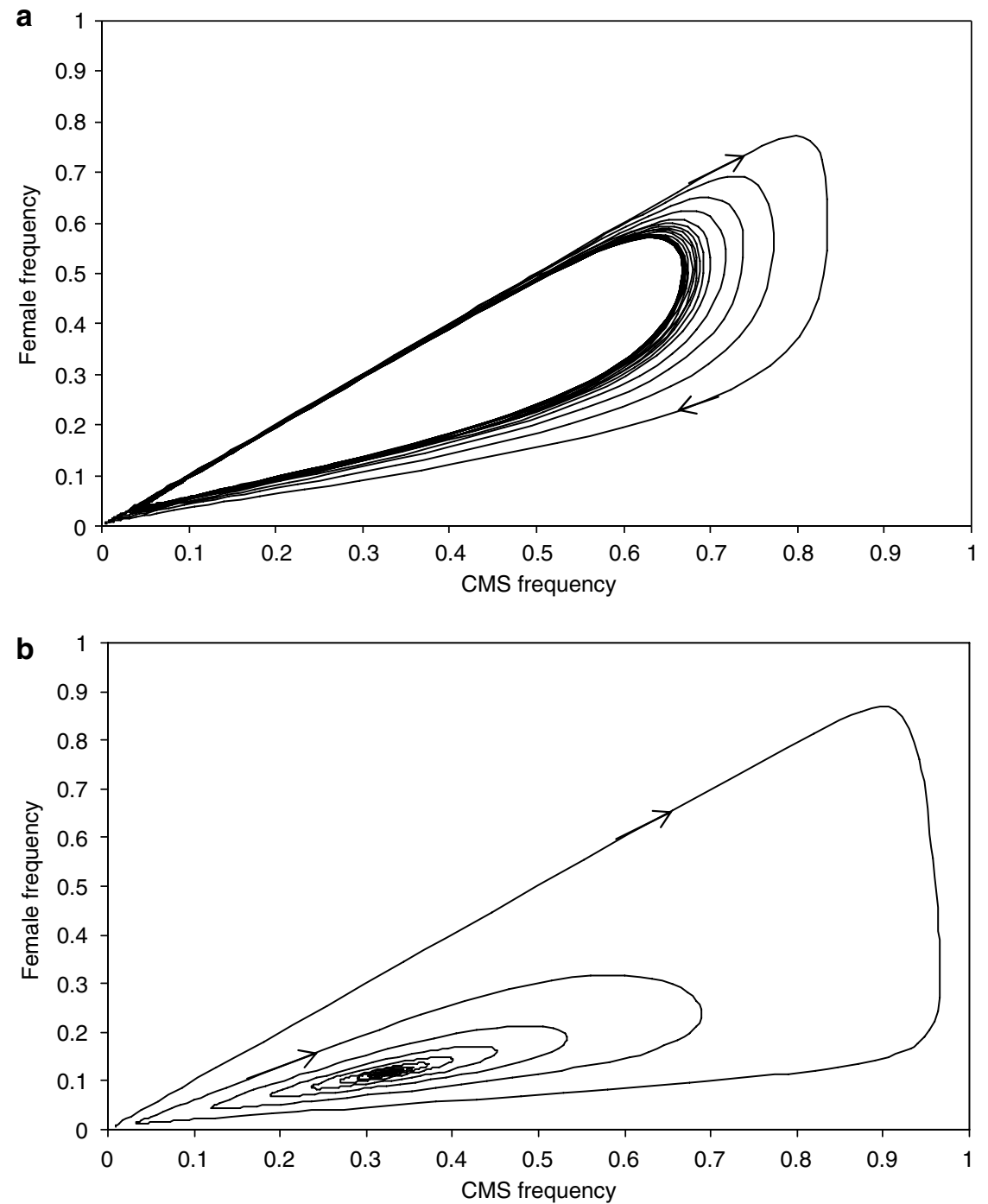

Figure 2 Examples of limit and dampened cycles. In both cases, the cost was dominant, constitutive and affected pollen. In case a $(F A=2.05$; $w_{\mathrm{s}}=0.55$ and $\left.c=0.25\right)$, CMS first increased in frequency, without being restored, which led to an increase in female frequency. When the restorer allele was selected, female frequency strongly decreased. At one point, CMS was not associated with fitter female phenotype, and thus decreased in frequency. As a result, one can observe dampened cycles that quickly converge towards a limit cycle. The exact same limit cycle was found regardless of the initial values in CMS and female frequencies. In case $\mathrm{b},\left(F A=2.2 ; w_{\mathrm{s}}=0.7\right.$ and $\left.c=0.2\right)$, a similar dynamics occurred but led to a dampened cycle. In the latter case, the point equilibrium was reached after 630 generations. 


\section{Female frequencies}

In all simulations that provided NC gynodioecy (all models except the model with expressed cost), we analysed the final female frequencies for the situations that presented a single equilibrium. When cost of restoration was constitutive, female frequency varied between 2 and 43\%; with a silent cost of restoration female frequency varied between 2 and 35\%. A linear regression (proc GLM, SAS, 2003) performed on these results revealed that both female fitness of male-sterile $F A^{*} w_{s}$ and the restoration cost values had a significant positive effect on the final female frequency in all models (for example, for dominant constitutive cost on pollen, effects of $F A^{*} w_{s}$ and cost were respectively $\mathrm{F}_{1,1326}=112$ $P<0.0001$ and $\left.\mathrm{F}_{1,1326}=5315 \quad P<0.0001\right)$. In the cases where limit cycles were found, the highest female frequency that was reached during the cycles varied from 5 to $80 \%$ among simulations. Again, the maximal female frequency increased significantly with female fitness (values of $A F^{*} w_{s}: \mathrm{F}_{1,1220}=113 P<0.0001$ ) and high restoration costs $\left(\mathrm{F}_{1,1220}=814 P<0.0001\right)$.

\section{Discussion}

The models show that selection can maintain gynodioecy with CMS and a male-fertile cytotype for a large set of parameter values, which contradicts the established idea that male-fertile cytotypes are difficult to maintain in gynodioecious species, without the benefit of selfpollination. In all cases of NC gynodioecy, the following phases were observed: (1) non-restored CMS (females) spread in the population, benefiting from a higher female fitness than 'normal' hermaphrodites; (2) when CMS have reached a sufficiently high frequency, genotypes carrying a restorer allele have a selective advantage (since they can produce pollen) and therefore increase in frequency; (3) as a direct consequence, female frequency decreases and, during this phase, CMS is increasingly carried by restored hermaphrodites; (4) if the male-fertile cytotype has sufficient female fitness (compared to the CMS cytotype), they increase in frequency and CMS frequency strongly decreases; and (5) because plants with both a male-fertile cytotype and a restorer allele express a cost of restoration, restorers are then counterselected and the population reverts back to a majority of plants having the male-fertile cytotype without restorer alleles.

These dynamics were allowed by one specific hierarchy of female fitness over genotypes, due to the combined action of CMS cost and female advantage. In addition, this also required a cost of restoration, which could be expressed in various terms: several possible hierarchies of male fitness over genotypes were then conceivable. We now discuss the biological interpretation of these fitness effects, as well as the implications for experimental studies that aim to measure fitness parameters in gynodioecious species.

\section{CMS cost and female advantage}

We found that for NC gynodioecy to be maintained, both a female advantage and a negative effect of CMS on female fitness are necessary, and these parameters must combine to give the highest female fitness to females and the lowest female fitness to restored hermaphrodites. Ross and Gregorius (1985), without specifically defining a CMS cost, also found that this hierarchy of female fitness was required to maintain CMS and male-fertile cytotypes in gynodioecious populations. The CMS cost we propose in this model played its role when a majority of male sterile cytotypes were restored (when the population contained few females), by conferring an advantage to hermaphrodites with male-fertile cytotypes over restored hermaphrodites. The male-fertile cytotype was thus selected when the restorer allele was common. This explains how male-fertile cytotypes can be maintained even if they never experience a female advantage nor benefit from self-pollination.

Biologically, a CMS cost would mean that cytotype identity could affect female fitness, which has indeed been shown in Plantago lanceolata (Van Damme, 1984) and Silene vulgaris (McCauley and Olson, 2003). The difference in fitness is expected to be greater between CMS and a 'normal' cytoplasm (than between CMS cytotypes). Indeed, the study of the molecular mechanism of CMS in cultivated species has revealed that most sterilizing genes arise de novo in plant mitochondria and result from intragenomic recombination, the expression of which is often constitutive in plant tissues (Budar et al., 2003; Hanson and Bentolila, 2004; Chase, 2007). Therefore, one might expect an additional cost associated with the constitutive expression of the sterilizing gene specific to the CMS cytoplasm. The experimental assessment of CMS cost will require CMS markers to discriminate between restored hermaphrodites and male-fertile hermaphrodites and compare their female fitness.

In addition, the model showed that female plants need to benefit from a female advantage that more than compensates for the CMS cost. Female advantage (as defined by the model) cannot be directly measured, since it is not possible to experimentally discriminate between the effects of the sexual phenotype and those of the cytotype when measuring fitness in female plants. However, what can be experimentally measured is the resulting female fitness $\left(F A^{*} w_{s}\right.$ in the present model) that can also be compared to the predictions of previous theoretical studies. Bailey et al. (2003) limited their study on female fitness to values between 1 and 2, but Gouyon et al. (1991) found gynodioecy for any value of female fitness higher than 1 . Here we found that the relative female fitness must exceed 1 but also be lower than 2, otherwise the CMS cytotype became fixed in the population leading to nuclear gynodioecy. Moreover, it must be noted that gynodioecy could be found with relative female fitness values only slightly higher than 1 . This is consistent with observations of Beta vulgaris ssp. maritima, for which no significant female advantage could be detected (Boutin et al., 1988). More generally, it could explain the lack of detectable female advantage in other gynodioecious species (Uno, 1982; Williams and Fenster, 1998; Alonso and Herrera, 2001).

Detecting opposite effects of cytotype (CMS cost) and sexual phenotype (female advantage) on female plants is possible if these effects do not act on the same plant traits. Indeed, if CMS cost occurs through newly arisen mitochondrial genes, one must expect that their effect differs from the result of female compensation (resource reallocation). This could indeed explain contradictory results obtained in Plantago maritima (Dinnetz and Jerling, 1997), in which females benefited from a higher 
Table 3 Hierarchies of the different genotypes for male and female fitness that allow nc gynodioecy, according to the type of cost of restoration (constitutive/silent, acting on female (seed)/male (pollen) fitness)

\begin{tabular}{|c|c|c|c|c|c|}
\hline \multirow[t]{2}{*}{ Type of cost of restoration } & & \multicolumn{2}{|c|}{ CMS } & \multicolumn{2}{|c|}{ Male fertile cytotype } \\
\hline & & No restorer & Active restorer & Silent restorer & No restorer \\
\hline \multirow{2}{*}{ Constitutive cost on female fitness } & Female fitness & $* * * *$ & * & $* *$ & $* * *$ \\
\hline & Male fitness & 0 & $* * *$ & $* * *$ & $* * *$ \\
\hline \multirow{2}{*}{ Constitutive cost on male fitness } & Female fitness & $* * * *$ & $* *$ & $* * *$ & $* * *$ \\
\hline & Male fitness & 0 & ** & $* *$ & $* * *$ \\
\hline \multirow[t]{2}{*}{ Silent cost on female fitness } & Female fitness & $* * * *$ & * & $* *$ & $* * *$ \\
\hline & Male fitness & 0 & $* * *$ & $* * *$ & $* * *$ \\
\hline \multirow[t]{2}{*}{ Silent cost on male fitness } & Female fitness & $* * * *$ & ** & $* * *$ & $* * *$ \\
\hline & Male fitness & 0 & $* * *$ & ** & $* * *$ \\
\hline
\end{tabular}

Abbreviation: CMS, cytoplasmic male sterility.

For a given type of cost, the highest number of (*) indicates the highest fitness. In the case of a constitutive cost, all hermaphrodites that have a restorer ensure the cost. For a silent cost, only the male fertile hermaphrodites bearing the restorer ensure the cost.

Considering female fitness, for any type of cost of restoration, only the situations for which females (CMS with no restorer) had the highest female fitness (female advantage) and the restored hermaphrodites had the lowest female fitness (CMS cost) allowed nc gynodioecy. Thus, when the cost of restoration affected female fitness, only restoration cost values permitting this hierarchy of female fitness could maintain nc gynodioecy.

number of seeds that could results from compensation, but produced seeds with a lower germination rate than hermaphrodites. This is classically interpreted as the result of a size-number trade-off in seeds, but one can consider that the lower germination rate could also be the result of a dysfunction due to sterilizing genes in the mitochondria.

\section{Cost of restoration}

We have shown that a cost of restoration must exist for NC gynodioecy to be maintained along with a malefertile cytotype. This possibility was previously suggested by Ross and Gregorius (1985), who did not explicitly define a cost of restoration, but by analysing various situations in terms of fitness hierarchies, identified one case for which this type of gynodioecy was stable; this case is similar to our case of a constitutive cost on female fitness, as seen by Delph et al. (2007).

Whereas most theoretical studies have focused on dominant costs of restoration, we showed that both dominant and recessive costs could maintain $\mathrm{NC}$ gynodioecy. As in Bailey et al. (2003) we found that the maintenance of $\mathrm{NC}$ gynodioecy occurred under a greater range of parameter values for recessive costs versus dominant restoration cost. Indeed, this provides a functional overdominance of the nuclear locus of restoration, and allows restorer alleles to be maintained in spite of very high costs. According to our results, we might expect to detect a cost of restoration only in homozygotes, which would require more complex experimental design for the detection of costs and could explain why experimental evidence for restoration cost is so rare. This is also consistent with the high level of heterozygosity at the restorer locus observed in hermaphrodites of gynodioecious Silene vulgaris (Bailey and McCauley, 2005).

Moreover, this model showed that NC gynodioecy could be maintained with a cost affecting either pollen or seed fitness. This had already been shown when two CMS co-occurred (Gouyon et al., 1991; Bailey et al., 2003) but was not known in case of male-fertile cytotype maintenance. Consequently, the hierarchy of fitness due to the cost of restoration, when NC gynodioecy is maintained, can show different patterns as summarized in Table 3. The common prediction drawn from the different scenarios is that individuals with male-fertile cytotypes and a silent restorer have a cost, causing them to be at a disadvantage compared to hermaphrodites that do not carry a restorer. One way to test this hypothesis empirically would be to obtain markers for restorer alleles in gynodioecious species. However, these are rarely available (Touzet et al., 2004). Alternatively, by indirectly estimating the number of restorers in genotypes through the results of reciprocal crosses, two experimental studies on pollen viability in Lobelia siphilitica (Bailey, 2002) and on seed quality for Plantago lanceolata (De Haan et al., 1997a) have suggested a cost for silent restorers. This is also consistent with our results showing that gynodioecy could be maintained with a cost affecting pollen or seed fitness. Finally, it should be noted that restoration costs on male or female fitness are not equivalent in terms of gynodioecy maintenance. A cost to female fitness allowed NC gynodioecy for a more restricted set of parameters, because it provided a disadvantage to the male-fertile cytotype. Here, restorers were selected, and had slowing the increase of CMS frequency, but male-fertile cytotypes with restorers had ensured a cost to female fitness and these plants could not compete with CMS individuals. CMS then went to fixation and only a nuclear polymorphism remained.

Our predictions differ from Bailey et al. (2003) regarding the nature of restoration cost that allow the maintenance of NC gynodioecy. Contrary to the case of two CMSs, we have shown that an expressed cost of restoration could not maintain both CMS and malefertile cytotypes. Indeed, when the cost is restricted to expressed restorers, restorers are neutral in the malefertile cytotype and their fate depends only on the CMS individuals. Thus the restorer allele reached an equilibrium frequency that depended on the relative female fertility of females and restored hermaphrodites, as in a pure nuclear gynodioecy; in that case, there was no frequency-dependent selection among the cytotypes. Consequently, the only way to explain the co-occurrence of male-fertile and CMS cytotypes in populations is that restorers have either a constitutive (acting on all 
categories of hermaphrodites) or a silent cost (acting only on hermaphrodites with male-fertile cytotypes).

When restoration (dominant) cost affected male fitness, NC gynodioecy was more frequently found with silent costs than with constitutive cost. It was even possible to maintain NC gynodioecy for cost values higher than 0.5 when restoration cost was silent (Figure 1). This can be explained by the fact that restored hermaphrodites did not have any cost. The probability for a restorer allele to be carried by a CMS individual, and therefore to be associated to a fitness advantage (restoration of male fertility, with no cost of restoration) increases with the CMS frequency in the population. This could explain why we found a positive linear relationship between the cost of restoration and the minimum value $F A^{*} w_{s}$ required for stable $\mathrm{NC}$ gynodioecy. In contrast, when restoration cost was constitutive, restored hermaphrodites also had the cost and thus the restorer allele could never be selected when the cost was too high. When there was a high cost to female fitness, for the same reason as above, restorers could be selected when the cost was silent. However, because individuals with male-fertile cytotype had a cost to their female fitness, they could not compete with CMS plants, leading to nuclear gynodioecy.

\section{Gynodioecy dynamics and female frequencies}

Gynodioecy with limit cycles was found in some of the calculations, in particular for dominant costs affecting pollen, which is similar to the results of previous studies that considered two CMS types co-occurring in a population (Bailey et al., 2003). Whether or not limit cycles do occur in populations is not known, because of a lack of suitable observations, but, as postulated by Gouyon et al. (1991) the high variation in female frequencies observed in some gynodioecious species could reflect such dynamics (for example, Dommée et al., 1978; Wolfe and Shmida, 1997; Graff, 1999; McCauley et al., 2000; Bailey, 2002; Asikainen and Mutikainen, 2003; Delph and Mutikainen, 2003; Barr, 2004; Murayama et al., 2004; Medrano et al., 2005). However, since our results showed that the approach of point equilibria was usually slow, even without a predicted limit cycle, populations displayed wide variations of female frequencies. Therefore, selection alone may explain high variation of female frequencies among populations in most of the cases that allow NC gynodioecy.

In both limit cycles and point equilibria, the predicted peak of female frequencies were associated with the value of $F A^{*} w_{s}$ (overall female fitness of male-sterile individuals). This prediction is consistent with studies based on species with low female frequencies in populations, for which weak or no difference has been found in female fitness between females and hermaphrodites (Uno, 1982; Williams and Fenster, 1998; Alonso and Herrera, 2001; Asikainen and Mutikainen, 2003).

\section{Conclusion}

This study has shown that selection can maintain malefertile cytotypes in gynodioecious populations through interactions among the seed fertility of cytotypes, the cost of restorer alleles and a female advantage. Despite the difficulty of experimentally testing for such complex effects, some published data on gynodioecious species
Gynodioecy and male-fertile cytoplasm

M Dufaÿ et al

suggest they may occur. Thus, this model provides an alternative set of theoretical predictions to compare with future studies on gynodioecious species, for which the exact genetic determinism is unknown. It also highlights the importance of considering all possible fitness differences among genotypes and phenotypes for both pollen and seeds in gynodioecious species. Finally, because it has been shown previously that metapopulation structure can strongly influence the conditions for gynodioecy maintenance (Pannell, 1997; Couvet et al., 1998), future theoretical studies should attempt to estimate the role of gene flow and genetic drift on the fate of male-fertile cytotypes in gynodioecious populations.

\section{Acknowledgements}

We thank Jean-François Arnaud, Marcel Dorken, Xavier Vekemans and two anonymous reviewers for their very helpful comments on various versions of the manuscript, as well as Licia Huffman-Touzet for proof-reading an earlier version of the manuscript. This work was supported by grants from the Région Nord-Pas de Calais and the European Community (European Regional Development Fund). MD also benefited from a BQR starting grant of the Université des Sciences et Technologies of Lille.

\section{References}

Alonso C, Herrera CM (2001). Neither vegetative nor reproductive advantages account for high frequency of malesteriles in southern Spanish gynodioecious Daphne laureola (Thymelaeaceae). Am J Bot 88: 1016-1024.

Asikainen E, Mutikainen P (2003). Female frequency and relative fitness of females and hermaphrodites in gynodioecious Geranium sylvaticum (Geraniaceae). Am J Bot 90: 226-234.

Bailey MF (2002). A cost of restoration of male fertility in a gynodiecious species, Lobelia syphilitica. Evolution 56: 21782186.

Bailey MF, Delph LF, Lively CM (2003). Modeling gynodioecy: novel scenarios for maintaining polymorphism. Am Nat 161: 762-776.

Bailey MF, McCauley DE (2005). Offspring sex-ratio under inbreeding and outbreeding in a gynodioecious plant. Evolution 59: 287-295.

Barr CM (2004). Soil mosture and sex ratio in a plant with nuclear-cytoplasmic sex inheritance. Proc $R$ Soc London B 271: 1935-1939.

Belhassen E, Dommée B, Atlan A, Gouyon PH, Pomente D, Assouad MW et al. (1991). Complex determination of male sterility in Thymus vulgaris L.: genetic and molecular analysis. Theor Appl Genet 82: 137-143.

Boutin V, Jean R, Valero M, Vernet P (1988). Gynodioecy in Beta maritima. Oecologia Plantarum 9: 61-66.

Brown GG (1999). Unique aspects of cytoplasmic male sterility and fertility restoration in Brassica napus. J Hered 90: 351-356.

Budar F, Touzet P, De Paepe R (2003). The nucleo-mitochondrial conflict in cytoplasmic male sterilities revisited. Genetica 117: 3-16.

Clifton SW, Minx P, Fauron CM, Gibson M, Allen JO, Sun H et al. (2004). Sequence and comparative analysis of the maize NB mitochondrial genome. Plant Physiol 136: 3486-3503.

Couvet D, Ronce O, Gliddon C (1998). Maintenance of nucleocytoplasmic polymorphism in a metapopulation: the case of gynodioecy. Am Nat 152: 59-70. 
Cuguen J, Wattier R, Saumitou-Laprade P, Forcioli D, Mörchen M, Van Dijk H et al. (1994). Gynodioecy and mitochondrial DNA polymorphism in natural populations of Beta vulgaris ssp maritima. Gen Sel Evol 26: S87-S101.

Charlesworth D (1981). A further study of the problem of the maintenance of females in gynodioecious species. Heredity 46: $27-39$.

Chase CD (2007). Cytoplasmic male sterility: a window to the world of plant mitochondrial-nuclear interactions. Trends Genet 23: 81-90.

Darwin C (1877). The Different Forms of Flowers on Plants of the Same Species. John Murray: London.

De Haan AA, Hundscheid MPJ, van Hinsberg A (1997a). Effects of CMS types and restorer alleles on plant performance on Plantago lanceola L.: an indication for cost of restoration. J Evol Biol 10: 803-820.

De Haan AA, Mateman AC, Van Dijk PJ, Van Damme JMM (1997b). New CMS types in Plantago lanceolata and their relatedness. Theor Appl Genet 94: 539-548.

Delannay X, Gouyon PH, Valdeyron G (1981). Mathematical study of the evolution of gynodioecy with cytoplasmic inheritance under the effect of a nuclear gene. Genetics 99: 169-181.

Delph LF, Mutikainen P (2003). Testing why the sex of the maternal parent affects seedling survival in a gynodioecious species. Evolution 57: 231-239.

Delph LF, Touzet P, Bailey MF (2007). Merging theory and mechanism in studies of gynodioecy. Trends Ecol Evol 22: $17-24$.

Dinnetz P, Jerling L (1997). Gynodioecy in Plantago maritima L.; no compensation for loss of male function. Acta Bot Neerl 46: 193-206.

Dommée B, Assouad MW, Valdeyron G (1978). Natural selection and gynodioecy in Thymus vulgaris L. Biol J Linn Soc 77: 17-28.

Fauron CMR, Casper M (1994). A second type of normal maize mitochondrial genome: an evolutionary link. Genetics 137 875-882.

Fénart S, Touzet P, Arnaud JF, Cuguen J (2006). Emergence of gynodioecy in wild beet (Beta vulgaris ssp. maritima L.): a genealogical approach using chloroplastic nucleotide sequences. Proc R Soc London B 273: 1391-1398.

Forcioli D, Saumitou-Laprade P, Valero M, Vernet P, Cuguen J (1998). Distribution of chloroplast DNA diversity within and among populations of the gynodioecious species Beta vulgaris ssp maritima (Chenopodiaceae). Mol Ecol 7: 1193-1204.

Frank SA (1989). The evolutionary dynamics of cytoplasmic male sterility. Am Nat 133: 345-376.

Gouyon PH, Vichot F, Van Damme JMM (1991). Nuclearcytoplasmic male sterility: single point equilibria versus limit cycles. Am Nat 137: 498-514.

Graff A (1999). Population sex structure and reproductive fitness in gynodioecious Sidalcea malviflora malviflora (Malvaceae). Evolution 53: 1714-1722.

Hanson MR, Bentolila S (2004). Interactions of mitochondrial and nuclear genes that affect male gametophyte development. Plant Cell 16: S154-S169.
Laporte V, Viard F, Béna G, Valero M, Cuguen J (2001). The spatial structure of sexual and cytonuclear polymorphism in the gynodioecious Beta vulgaris ssp maritima: I/ at a local scale. Genetics 157: 1699-1710.

Lewis D (1941). Male sterility in natural populations of hermaphrodite plants: the equilibrium between females and hermaphrodites to be expected with different types of inheritance. New Phytol 40: 56-63.

McCauley DE, Olson MS (2003). Associations among cytoplasmic molecular markers, gender, and components of fitness in Silene vulgaris, a gynodioecious plant. Mol Ecol 12: 777-787.

McCauley DE, Olson MS, Emery SN, Taylor DR (2000). Population structure influences sex ratio evolution in a gynodioecious plant. Am Nat 155: 814-819.

McCauley DE, Taylor DR (1997). Local population structure and sex-ratio: evolution in gynodioecious plants. Am Nat 150: 406-419.

Medrano M, Alonso C, Herrera CM (2005). Mating system, sex ratio, and persistence of females in the gynodioecious shrub Daphne laureola L. (Thymelaeceae). Heredity 94: 37-43.

Murayama K, Yahara T, Terachi T (2004). Variation of female frequency and cytoplasmic male-sterility gene frequency among natural gynodioecious populations of wild radish (Raphanus sativus L.). Mol Ecol 13: 2459-2464.

Olson MS, McCauley DE (2002). Mitochondrial DNA diversity, population structure, and gender association in the gynodioecious plant Silene vulgaris. Evolution 56: 253-262.

Pannell J (1997). The maintenance of gynodioecy and androdioecy in a metapopulation. Evolution 51: 10-20.

Ross MD, Gregorius HR (1985). Selection with gene-ctyoplasm interactions. II. Maintenance of gynodioecy. Genetics 109: 427-439.

SAS (2003). SAS Institute Inc. release 9.1. Cary, NC, USA.

Städler T, Delph LF (2002). Ancient mitochondrial haplotypes and evidence for intragenic recombination in a gynodioecious plant. Proc Natl Acad Sci USA 99: 11730-11735.

Tang VH, Pring DR, Muza FR, Yan B (1996). Sorghum mitochondrial orf25 and a related chimeric configuration of a male-sterile cytoplasm. Curr Genet 29: 265-274.

Touzet P, Hueber N, Bürkholz A, Barnes S, Cuguen J (2004). Genetic analysis of male fertility restoration in wild cytoplasmic male sterility G of beet. Theor Appl Genet 109: 240-247.

Uno GE (1982). Comparative reproductive biology of hermaphroditic and male-sterile Iris douglasiana Herb. (Iridaceae). Am J Bot 69: 818-823.

Van Damme JMM (1984). Gynodioecy in Plantago lanceolata L. III. Sexual reproduction and the maintenance of male steriles. Heredity 52: 77-93.

Van Damme JMM, Hundscheid MPJ, Ivanovic S, Koelewijn HP (2004). Multiple CMS-restorer gene polymorphism in gynodioecious Plantago coronopus. Heredity 93: 175-181.

Williams HL, Fenster CB (1998). Ecological and genetic factors contributing to the low frequency of male sterility in Chamaecrista fasciculata (Fabaceae). Am J Bot 85: 1243-1250.

Wolfe LM, Shmida A (1997). The ecology of sex expression in a gynodioecious israeli desert shrub (Ochradenus baccatus). Ecology 78: 101-110. 